\title{
Commentary on Martin \& Pacherie. Out of nowhere: Thought insertion, ownership and context-integration
}

\section{Max Seeger}

This is a preprint version. For citation, please refer to the published version: Consciousness and Cognition 22 (2013), 261-263.

In their article "Out of nowhere: Thought insertion, ownership and context-integration" (2013), Jean-Remy Martin \& Elisabeth Pacherie propose a novel approach to the explanation of thought insertion: the context-integration approach. Since the article contains many novel and important ideas, it is the more regrettable that it suffers from considerable conceptual confusions which result in a misled criticism of the standard approach. Contrary to what the authors claim, their own view can be construed as a refined version of the standard approach. The main problem with their discussion is an equivocation on the term 'sense of ownership'. Moreover, they fail to recognize an ambiguity in the term 'sense of agency'. Since the sketchiness of these two notions constitutes a shortcoming of the debate in general, I seize Martin \& Pacherie's paper as an opportunity to do some conceptual housekeeping.

According to the standard approach, subjects of inserted thoughts lack a sense of agency (SoA) for the inserted thought, but retain a sense of ownership (SoO). Martin \& Pacherie argue-purportedly in opposition to the standard approach-that subjects of inserted thoughts lack both a SoA and a SoO for the thought. They hold that the lacking SoA is but a consequence of the missing SoO which constitutes the core of thought insertion.

Further, they propose a novel explanation of why the SoO is missing in thought insertion. Based on plenty of empirical findings, they argue that schizophrenic subjects fail to integrate contextual information about the causes of a given thought with the thought itself. This lack of integration leads to the thought's a decontextualized phenomenology and its appearing to come out of nowhere.

Martin \& Pacherie criticize the standard approach for attributing an intact SoO to subjects of inserted thoughts and claim that thought insertion fundamentally involves an abnormal SoO. This criticism is based on a serious misunderstanding of what the standard approach actually says. When proponents of the standard approach ${ }^{1}$ claim that subjects have an intact SoO they either mean that subjects have introspective access to the thought and are conscious of it, or they mean that subjects experience the thought as within their own subjectivity. ${ }^{2}$

Proust defines the SoO as "a sense of this thought as being the one that is presently occupying one's attention" (2009, p. 253). Gallagher defines the SoO as the "sense that it is I who am experiencing the [...] thought" (2004, p. 9). According to Young, "the

\footnotetext{
${ }^{1}$ With 'proponents of the standard approach' I primarily refer to those authors (and works) mentioned by Martin \& Pacherie: Stephens \& Graham (2000), Gallagher (2004), Peacocke (2007), Young (2008), Proust (2009), and Sousa \& Swiney (2011).

${ }^{2}$ Cf. also Sousa \& Swiney's (2011) distinction between two notions of SoO.
} 
patient recognises that her putatively alien thoughts are thoughts that occur within her own psychological boundaries, and that the experience is therefore her experience (she has a sense of ownership)" (2008, p. 850; Young's emphasis). Sousa \& Swiney define the SoO as "the sense that the thoughts that I introspect occur within the boundaries of my subjectivity [...] that they are part of my stream of consciousness." They state, accordingly, that "there is a normal sense of thought ownership in thought insertion: the patients experience the thoughts as occurring in their subjectivity." (2011, n.p.) Finally, Stephens \& Graham's claim that a subject has an intact sense of subjectivity (they do not use the term 'ownership') is meant to say that "[s]he admits to being the subject in whom the thought occurs" (2000, p. 153).

Surprisingly, Martin \& Pacherie deny none of these claims. What, then, do they claim, when they say-purportedly in opposition to the standard approach-that subjects lack a SoO? The passage that comes closest to providing a definition is unfortunately not very illuminating: the SoO is characterized as "the feeling or sense that our thoughts belong to us". Does that mean that whenever a subject sincerely claims to experience a thought as not her own, then this claim expresses a missing SoO? Martin \& Pacherie seem to assume such a view. ${ }^{3}$ But that's not a viable definition. There are different ways in which a thought can be experienced as one's own and we distinguish between SoO and SoA precisely to unravel this ambiguity (cf. Campbell 2002, p.36; Stephens \& Graham 2000, p. 152). Hence, defining the SoO simply as the sense that a thought is one's own won't do.

We get a better grip on what Martin \& Pacherie have in mind when we look at the phenomenon of causal coherence which is supposed to underlie or accompany the SoO. ${ }^{4}$ The feeling of causal coherence arises when information regarding "the causal factors that trigger and/or constrain" (p. 115) episodes of thinking is integrated with the thought. If the causal-contextual information is not integrated properly, the thought does not feel causally coherent and the subject lacks a SoO for the thought. Thoughts that are causally coherent are experienced as "coherent and unified episodes of thinking" (p. 115); they have "a specific phenomenology of coherence" (p. 116), and "[their presence] within our stream of consciousness will feel 'natural' and normal" ( $p$. 116). In contrast, thoughts that lack causal coherence appear "decontextualized", "abnormal, incoherent and fragmented" (p. 115), as if "coming out of nowhere" (p. 121). Martin \& Pacherie's claim that subjects lack a SoO thus says that inserted thoughts have a fragmented phenomenology.

If this is what they mean, however, the standard approach's claim that subjects have an intact SoO is fully compatible with Martin \& Pacherie's claim that subjects lack a SoO. Proponents of the standard approach do not deny that thought insertion involves a fragmented phenomenology, and Martin \& Pacherie do not deny that inserted thoughts are experienced as within the subject's stream of consciousness or subjectivity. The first aspect of Martin \& Pacherie's critique is therefore unfounded.

The second aspect of Martin \& Pacherie's critique concerns the sense of agency (SoA). They agree with the standard approach's claim that subjects lack a SoA, but contend that this is not what's at the core of thought insertion; subjects essentially lack the SoO,

\footnotetext{
${ }^{3}$ They argue from the fact that subjects report inserted thoughts as not their own to the conclusion that subjects lack a SoO.

${ }^{4}$ It is not clear, whether causal coherence is an additional feeling that underlies the SoO or whether they are one and the same.
} 
and the lacking SoA is but a consequence of that. The standard approach, their critique holds, focuses on the wrong phenomenon.

When proponents of the standard approach claim that subjects lack a SoA for inserted thoughts, they mean that subjects do not have a feeling of bringing the thoughts about deliberately, voluntarily, or intentionally. We have a SoA for thoughts when we deliberately turn our minds to a specific task or topic; we don't have a SoA for thoughts that come to mind unasked and involuntary (unbidden thoughts). To describe the lacking SoA in inserted thoughts, some proponents of the standard approach in fact point to the phenomenology of unbidden thoughts. ${ }^{5}$

Martin \& Pacherie criticize that the standard approach cannot distinguish the phenomenology of inserted thoughts from that of everyday unbidden thoughts, for which we don't have a SoA either. ${ }^{6}$ However, the phenomenology of inserted thoughts, they assume, is essentially different from that of unbidden thoughts. Describing thought insertion as involving a lacking SoA thus doesn't capture what's at the heart of the matter.

In response to similar worries, Sousa \& Swiney (2011) propose a refined version of the standard approach. They distinguish two senses of agency: a sense of intentionally guiding a thought (henceforth: sense of intentional agency) and a sense of causally generating a thought (henceforth: sense of causal agency). The sense of causal agency is the broader phenomenon and accompanies not only to intended thoughts, but also unbidden thoughts. ${ }^{7}$ According to Sousa \& Swiney, subjects of inserted thoughts lack a sense of causal agency and not merely a sense of intentional agency (as other proponents of the standard approach hold). This distinction provides the answer to Martin \& Pacherie's second criticism: the difference between inserted thoughts and unbidden thoughts is that in inserted thoughts subjects lack a sense of causal agency, in unbidden thoughts subjects lack a sense of intentional agency.

Martin \& Pacherie's context-integration approach is not only compatible with, but strongly resembles Sousa \& Swiney's version of the standard approach. The essential claim of both views is that subjects do not experience themselves as the causal originators of inserted thoughts. I hope these considerations place the contextintegration approach more adequately within the ongoing debate. Since my discussion concerns the descriptive part only, the originality of the explanatory approach stands unquestioned.

\footnotetext{
${ }^{5}$ See Stephens \& Graham 2000, pp. 150f.; Gallagher 2004, p. 9; Peacocke 2007, p. 368. According to Proust, having a SoA involves "intending to think this particular thought" $(2009,253)$ and Young equates the SoA with the sense of initiating a thought $(2008$, p. 853).

${ }^{6}$ Langland-Hassan (2008, p. 371) makes a similar observation, but reaches a different conclusion.

${ }^{7}$ Note that Martin \& Pacherie define the SoA as "the feeling or sense that we are the causal generator of our thoughts". This sounds as if they have the sense of causal agency in mind. But their discussion reveals that they really have the sense of intentional agency in mind. We find similar discrepancies (between literal definition and actual use of the term 'SoA') with many other authors, including the proponents of the standard approach (see e.g. Gallagher 2004, p. 9; Young 2008, p. 850).
} 


\section{References}

Campbell, J. (2002). The ownership of thoughts. Philosophy, Psychiatry, \& Psychology, 9, 35-39.

Gallagher, S. (2004). Neurocognitive models of schizophrenia: a neurophenomenological critique. Psychopathology, 37, 8-19.

Langland-Hassan, P. (2008). Fractured phenomenologies: thought insertion, inner speech, and the puzzle of extraneity. Mind \& Language, 23, 369-401.

Martin, J.-R., \& Pacherie, E. (2013). Out of nowhere: Thought insertion, ownership and contextintegration. Consciousness and Cognition, 22, 111-122.

Peacocke, C. (2007). Mental action and self-awareness (I). In B. McLaughlin, \& J. Cohen (Eds.), Contemporary debates in the philosophy of mind (pp. 358-376). Oxford: Blackwell.

Proust, J. (2009). Is there a sense of agency for thought? In L. O'Brien, \& M. Soteriou (Eds.), Mental Action (pp. 253-279). Oxford: Oxford University Press.

Stephens G.L., \& Graham G. (2000). When Self-Consciousness Breaks: Alien Voices and Inserted Thoughts. Cambridge: MIT Press.

Sousa, P., \& Swiney, L. (2011). Thought insertion: Abnormal sense of thought agency or thought endorsement? Phenomenology and the Cognitive Sciences DOI 10.1007/s11097-0119225-z

Young, G. (2008). On how a child's awareness of thinking informs explanations of thought insertion. Consciousness and Cognition, 17, 848-62. 